\title{
Successful unrelated donor cord blood transplantation for Glanzmann's thrombasthenia
}

Kitko CL, Levine JE, Matthews DC, Carpenter PA. Successful unrelated donor cord blood transplantation for Glanzmann's thrombasthenia. Pediatr Transplantation 2009. (C) 2009 John Wiley \& Sons A/S.

Abstract: GT, a rare disorder of platelet function, can lead to lifethreatening bleeding, particularly following the development of antiplatelet antibodies. Curative therapy includes HCT but previous reports are limited predominantly to matched siblings and have excluded CBT. Delayed or non-engraftment of platelets because of antiplatelet antibodies might be particularly concerning after CBT for GT. Here, we report two successful unrelated cord blood transplants for GT. Recurrent life-threatening bleeding was the primary indication for HCT, with one patient developing antiplatelet antibodies preHCT. Bleeding risks associated with delivery of the conditioning regimen and the toxicity that follows should be carefully considered, including tunneled central venous line catheter placement, inclusion of B cell-specific therapy to potentially decrease antiplatelet antibody production, and targeted busulfan dosing. This is the first report of successful unrelated cord blood HCT for GT and indicates that modifications to supportive care can improve the safety of this potentially curative therapy for patients with severe, life-threatening disease manifestations.
Carrie L. Kitko ${ }^{1}$, John E. Levine ${ }^{1}$, Dana
C. Matthews ${ }^{2}$ and Paul A. Carpenter

${ }^{1}$ Department of Pediatrics and Internal Medicine, Blood and Marrow Transplant Program, University of Michigan, Ann Arbor, MI, ${ }^{2}$ Department of Pediatrics, University of Washington, Seattle, WA, ${ }^{3}$ Fred Hutchinson Cancer Research Center, Seattle, WA, USA

Key words: Glanzmann's - thrombasthenia platelets - cord blood - transplantation

Paul A. Carpenter, Fred Hutchinson Cancer Research Center, Mailstop D5-290, 1100 Fairview Avenue N, Seattle, WA 98109-1024, USA

Tel.: +206 6675191

Fax: +206 6671024

E-mail: pcarpent@fhcrc.org

Accepted for publication 4 September 2009
GT is an autosomal recessive disorder characterized by variable absence or dysfunction of platelet GPIIb/IIIa. Fatal hemorrhages may occur in severe cases because bridging of GPIIa/IIIb complexes with fibrinogen is required for primary hemostasis. Marrow transplantation from HLA-matched siblings, and less commonly from unrelated donors, has been curative but is reserved for severe cases, especially when alloim-

\footnotetext{
Abbreviations: ADP, adenosine 5'-diphosphate; BMT, bone marrow transplantation; BUCY, busulfan and cyclophosphamide; $\mathrm{CMV}$, cytomegalovirus; $\mathrm{CVC}$, central venous catheter; CBT, cord blood transplantation; FITC, fluorescein isothiocyanate; GAM-FITC, goat anti-mouse-fluorescein isothiocyanate; GPIIb/IIIa, glycoproteins IIb/IIIa; GT, Glanzmann's thrombasthenia; GVHD, graft versus host disease; HCT, hematopoietic stem cell transplantation; HLA, human leukocyte antigen; ITP, immune thrombocytopenic purpura; IVIG, intravenous gammaglobulin; PICC, peripherally inserted cutaneous catheter; rfVIIa, recombinant human-activated factor VII; TNC, total nucleated cell.
}

munization has rendered platelet transfusions ineffective (1-8). Paradoxically, life-threatening hemorrhage may be precipitated during the peritransplant period, especially when antiGPIIb/IIIa antibodies coincide with routine procedures including CVC placement or conditioning-associated mucosal disruption. Our report details two cord blood transplants for GT.

\section{Patients, materials, and methods}

Pretransplant characteristics, preparative regimens, and outcomes are summarized in Table 1. Supportive care followed institutional standard practices and included ursodeoxycholic acid on Day -1 (D-1) through D + 90; IVIG therapy; chemoprophylaxis for Pneumocystis jirovecii, varicella-zoster virus, and yeast; and antiviral therapy for CMV.

Platelet GPIIb/IIIa expression levels were analyzed by flow cytometry. Whole blood from normal controls and Patient 2 was collected in EDTA and centrifuged at $300 \mathrm{~g}$ for $10 \mathrm{~min}$. 


\section{Kitko et al.}

Table 1. Demographics and outcomes of CBT recipients

\begin{tabular}{|c|c|c|}
\hline & Patient 1 & Patient 2 \\
\hline Age at transplant, yr & 9.0 & 4.0 \\
\hline Race & White & White \\
\hline Clinical indications for UCBT & Life-threatening hemorrhages & Life-threatening hemorrhages \\
\hline Pretransplant therapies & $\begin{array}{l}\text { Platelet and red cell transfusions, } \\
\epsilon \text {-aminocaproic acid, rfVIla, nasal cautery }\end{array}$ & $\begin{array}{l}\text { Platelet and red cell transfusions, } \\
\epsilon \text {-aminocaproic acid, rfVlla }\end{array}$ \\
\hline HLA-matching & $10 / 10^{*}$ & $5 / 6$ \\
\hline Mismatch locus & - & A antigen \\
\hline \multicolumn{3}{|l|}{ Cell dose (post-thaw) } \\
\hline TNCs $\times 10^{7} / \mathrm{kg}$ & 13 & 8.0 \\
\hline $\mathrm{CD} 34 \times 10^{5} / \mathrm{kg}$ & 2.0 & 9.4 \\
\hline Central venous access & Double lumen Hickman & PICC line \\
\hline \multicolumn{3}{|l|}{ Preconditioning } \\
\hline Rituximab & No & Yes $^{\dagger}$ \\
\hline \multicolumn{3}{|l|}{ Conditioning } \\
\hline Busulfan (mg/kg) & $16^{\ddagger}$ & $16^{\ddagger}$ \\
\hline Cyclophosphamide (mg/kg) & $200^{\ddagger}$ & $200^{*}$ \\
\hline ATGAM $(\mathrm{mg} / \mathrm{kg})$ & $90^{+}$ & $90^{+}$ \\
\hline Post-transplant bleeding prophylaxis & None & $\epsilon$-aminocaproic acid \\
\hline \multicolumn{3}{|l|}{ Graft vs. host disease (GVHD) prophylaxis } \\
\hline Tacrolimus & $D-3$ to $+100^{\S}$ & $\mathrm{D}-1$ to $+80^{\circ}$ \\
\hline Mycophenolate mofetil & DO to $+35^{* *}$ & D0 to $+35^{* *}$ \\
\hline \multicolumn{3}{|l|}{ Days to engraftment } \\
\hline Neutrophils ${ }^{\dagger \dagger}$ & 26 & 23 \\
\hline Platelets & 48 & 31 \\
\hline \multicolumn{3}{|l|}{ Transfusions post-transplant admission } \\
\hline Red cells, Units & 14 & 5 \\
\hline Last red cell transfusion & $\mathrm{D}+89$ & $\mathrm{D}+51$ \\
\hline Platelets, Units & $54^{\S \S} / 24^{\bullet \bullet}$ & $5^{\S \S} / 10^{\bullet \oplus}$ \\
\hline Last platelet transfusion & $\mathrm{D}+41$ & $\mathrm{D}+31$ \\
\hline Follow-up, months & 45 & 12 \\
\hline \multicolumn{3}{|l|}{ Donor chimerism, \% } \\
\hline T cell (CD3) & - & 100 \\
\hline Myeloid (CD33) & - & 100 \\
\hline Whole blood & 100 & - \\
\hline \multicolumn{3}{|l|}{ GVHD } \\
\hline Acute (Grade I-IV) & None & $\|$ \\
\hline Chronic (NIH global severity) & None & None \\
\hline Initial hospital discharge day & 40 & 26 \\
\hline \multicolumn{3}{|l|}{ Antiplatelet antibodies } \\
\hline Pretransplant & Negative & Positive \\
\hline Post-transplant & - & Negative \\
\hline \multicolumn{3}{|l|}{ Platelet GPIIb/IIla flow cytometry } \\
\hline \multicolumn{3}{|l|}{ Pretransplant } \\
\hline Normal (GAM-FITC, $n=5$ ), range & - & $1.66,1.12-2.55$ \\
\hline Patient (GAM-FITC) & - & 1.31, range not applicable \\
\hline Normal ( $\alpha$ llb/IIla-FITC), range & - & 189, 166-219 \\
\hline Patient ( $\alpha$ llb/IIla-FITC) & - & 6.98 , range not applicable \\
\hline \multicolumn{3}{|l|}{ Post-transplant } \\
\hline Normal (GAM-FITC, $n=4)$, range & - & $2.00,1.36-2.94$ \\
\hline Patient (GAM-FITC) & - & 2.27, range not applicable \\
\hline Normal ( $\alpha$ llb/IIla-FITC), range & - & - \\
\hline Patient ( $\alpha$ llb/llla-FITC) & - & 491, range not applicable \\
\hline
\end{tabular}

*High resolution confirmatory HLA typing on both patient and cord blood unit were obtained.

†Rituximab $375 \mathrm{mg} / \mathrm{m}^{2}$ on $\mathrm{D}-10$.

Fintravenous busulfan (D-9 to D-6) targeted to a steady state concentration of 750-850 ng/mL, Cyclophosphamide (D-5 to D-2). Equine antithymocyte globulin (D-4 to D-2).

${ }^{\S}$ Tacrolimus continuous infusion from D-3 and then orally administered in two divided doses from D+34 targeted to a serum trough concentration of 10-15 $\mathrm{mg} / \mathrm{mL}$. "Tacrolimus continuous infusion from D-1 and then orally administered in three divided doses from D+20 targeted to a serum trough concentration of 5-10 $\mathrm{ng} / \mathrm{mL}$.

${ }^{* *}$ Mycophenolate mofetil $15 \mathrm{mg} / \mathrm{kg}$ three times daily from D0 and then twice daily from D+35.

${ }^{\dagger}$ Neutrophil engraftment was defined as the first of three consecutive days when ANC exceeded 500/ $\mu \mathrm{L}$.

HPlatelet engraftment was defined as the first of three consecutive days when the platelet count exceeded $20000 / \mu \mathrm{L}$, and at least one week after the last transfusion.

${ }^{\S}$ P Pooled platelets.

" Single donor platelets. 
Platelet rich plasma was removed and centrifuged at $1550 \boldsymbol{g}$ for seven minutes. The platelet pellet was adjusted to a concentration of $300 \times 10^{6}$ platelets per $\mathrm{mL}$, and $100 \mu \mathrm{L}$ of this suspension was incubated for $15 \mathrm{~min}$ at $37^{\circ} \mathrm{C}$ with saturating concentrations of murine antihuman GPIIb/IIIa antibody (AMAC Inc, Westbrook, ME, USA), washed twice, and resuspended. Platelets were then incubated with FITC conjugated goat anti-mouse antibody (BD Biosciences, San Jose, CA, USA) for $15 \mathrm{~min}$ at room temperature, washed twice and analyzed on a FACS scan (BD Biosciences). Platelets were gated based on their forward and side scatter characteristics, and FITC mean fluorescence intensity was recorded using histogram plots.

\section{Case 1}

Patient 1 presented with prolonged bleeding after circumcision and both bleeding and bruising after immunizations. GT was diagnosed at age 22 months based on platelet aggregation studies, which revealed a pathognomonic pattern of normal aggregation to ristocetin but absent response to ADP, epinephrine, and collagen. Flow cytometry analysis at a research laboratory also found no evidence of fibrinogen receptor components, consistent with the diagnosis of Type I GT. He received only three platelet transfusions through age five yr but almost 20 platelet transfusions by age eight. He was hospitalized five times for bleeding from nasal and gastrointestinal mucosae, or into joints. AntiGPIIb/IIIa antibodies did not emerge but unrelated cord blood transplantation was considered given the recurrent life-threatening bleeds and two presentations with hemorrhagic shock. Cord blood was chosen over an adult volunteer donor given the excellent level of matching, the large cell dose, and the reduced potential for GVHD. Placement of a tunneled CVC for transplant was complicated by hypovolemic shock because of a massive hemothorax that was evacuated via a thoracotomy tube. Hemostasis was achieved using frequent platelet transfusions and rfVIIa $300 \mathrm{mcg} / \mathrm{kg}$ every six h for $24 \mathrm{~h}$. Initiation of transplant conditioning was delayed for six days to allow for recovery and was well tolerated. Bilateral serosanguinous pleural effusions on $\mathrm{D}+7$ required drainage over the subsequent two weeks through thoracotomy tubes placed again under the cover of rfVIIa and platelet transfusions. Although an etiology for the effusions was not established, an inflammatory or capillary leak response to the transplant conditioning regimen in the context of a resolving hemothorax was considered plausible because pleural fluid cultures remained negative, and absence of frank blood was inconsistent with the diagnosis of recurrent hemothorax. Platelets were transfused to maintain platelet counts above $20000 / \mu \mathrm{L}$ or prevent bleeding. Transfusion frequency was increased as necessary around the time of invasive procedures. Neutrophil and platelet engraftment occurred on $\mathrm{D}+26$ and $\mathrm{D}+48$, without further bleeding episodes. Episodes of asymptomatic CMV reactivation on $\mathrm{D}+40$ and $\mathrm{D}+167$ were treated successfully with valganciclovir and $\mathrm{CMV}$ immune globulin. $\mathrm{He}$ did not develop GVHD, and tacrolimus was discontinued by $\mathrm{D}+250$. At 45 months after transplant, he was a straight A student in middle school, growing normally and unrestricted in his activities with a Lansky performance score of $100 \%$. He was taking only levothyroxine for hypothyroidism that developed $2.5 \mathrm{yr}$ after transplant. The absence of recurrent bleeding episodes that followed recovery of normal platelet counts and full donor chimerism was an acceptable surrogate for normal platelet function. Formal platelet function studies or GPIIb/IIIa expression levels were not obtained.

\section{Case 2}

Patient 2 presented in infancy with petechial rashes, prolonged oozing from immunization sites, and bruising as his mobility increased. Platelet count and coagulation studies were normal, but Platelet GPIIb/IIIa expression was significantly reduced (Table 1 and Fig. 1). Subsequent recurrent mucosal bleeds included three life-threatening hemorrhages between ages 18 and 21 months that were managed with absorbable gelatin-compressed sponge packs, $\epsilon$-aminocaproic acid, rfVIIa, transfused red cells, and platelets. By three years of age, platelet

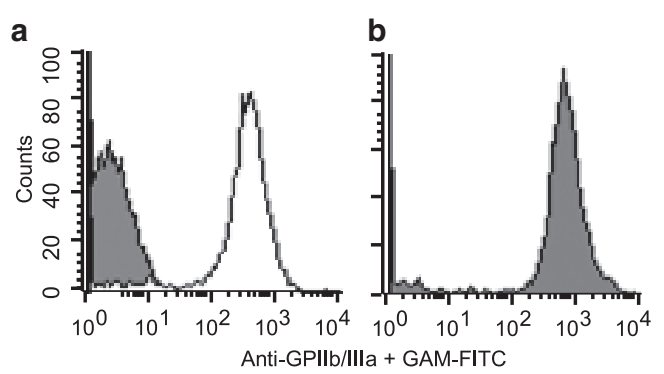

Fig. 1. Platelet glycoprotein IIb/IIIa expression before and after transplant. Platelet GPIIb/IIIa expression levels are shown before transplant (a) for Patient 2 (dark gray) compared to a representative normal control (white) and after transplant (b) for Patient 2 (normal control not shown). 
transfusions were ineffective because of antiGPIIb/IIIa antibodies and, in the absence of an appropriate marrow donor, he was evaluated for unrelated cord blood transplantation. A PICC was placed on D-19 for conditioning, which included one dose of rituximab on D-10. Bleeding prophylaxis throughout the period of mucositis included $\epsilon$-aminocaproic acid $15 \mathrm{mg} / \mathrm{kg} \mathrm{IV}$ every six hours beginning D-3. Nonetheless, bleeding from the nasal and oral mucosae developed on D0, requiring balloon tamponade, rfVIIa $90 \mathrm{mcg} / \mathrm{kg}$ every two hours (three doses), and platelets two units every $4 \mathrm{~h}$ for $24 \mathrm{~h}$. Further bleeding was prevented by resumption of $\epsilon$-aminocaproic acid therapy at $45 \mathrm{mg} / \mathrm{kg}$ every six hours until neutrophil engraftment on $\mathrm{D}+23$ (platelet count $35 \times 10^{9} / \mathrm{L}$ ). CMV viremia on $\mathrm{D}+6$ was treated with foscarnet and subsequently valganciclovir. Transient BK viruria with hematuria and frequent dysuria were treated with transfusions, phenazopyridine, and oxybutynin. The PICC line required replacement twice in the first two months (once for self-removal, once for thrombus and bacteremia), but otherwise was suitable for all intravenous needs. Mild Grade II GVHD of the stomach on D+51 was treated with prednisone and mycophenolate mofetil that were sequentially discontinued by $\mathrm{D}+265$. At 344 days after transplant antiplatelet antibodies were absent. Platelet GPIIa/IIIb expression was normal (Fig. 1). As there was no evidence of GVHD, a tacrolimus taper was begun. Now, 12 months post-HCT, he has no evidence of bleeding, normal blood counts, growth, and development, and a Lansky performance score of $100 \%$.

\section{Discussion}

To our knowledge, this is the first report of cord blood transplantation for GT, and several practical considerations are worth comment. First, the risk of placing a tunneled CVC in patients who are refractory to platelet transfusions cannot be underestimated. At the time of CVC placement for the 14 reported patients, five fortunately remained responsive to platelet transfusions (1-8). Among the remaining nine patients, only four were clearly stated to be refractory to platelet transfusions, and rfVIIa was the mainstay for achieving hemostasis in this group $(1,6,8,9)$. Bleeding in our Patient 2 had been only minimally responsive to rfVIIa. A near fatal hemorrhage associated with placement of a tunneled CVC in Patient 1, together with the knowledge of a similar incident in another patient, prompted us to select a PICC line for
Patient 2 prior to conditioning. This PICC line was replaced twice post-transplant but otherwise allowed one potential iatrogenic risk for major pretransplant hemorrhage to be avoided.

Our next decision to administer pretransplant rituximab to Patient 2 attempted to abrogate antiplatelet antibodies. Rituxumab has been used to treat refractory ITP in children (10), and we were quite familiar with using standard $375 \mathrm{mg} / \mathrm{m}^{2}$ doses of rituximab to treat refractory chronic GVHD and/or immune-mediated cytopenias after $\operatorname{HCT}(11,12)$. We reasoned that the risk to benefit ratio of a single dose of rituximab added to the conditioning regimen was likely to be favorable when faced with the known risks for delayed engraftment or non-engraftment of platelets after cord blood transplantation. There are no published data on rituximab being used for antibody desensitization before HCT. However, alloantibody desensitization protocols that include rituximab before renal transplantation have been successful in preventing antibodymediated renal allograft rejection $(13,14)$ although failure to completely eradicate alloantibodies may still occur because rituximab does not deplete plasma cells $(13,15)$.

Intense cytotoxic conditioning appears to have controlled this problem in the small number of cases where platelet alloimmunization was present, but rituximab offers an additional and more targeted $\mathrm{B}$ cell approach to reducing alloantibodies. It is of interest that two reports included alemtuzumab with conditioning, which also achieves prolonged host B cell depletion $(7,8)$. One patient who did not receive anti-B cell serotherapy developed anti-GPIIb/ IIIa antibodies on D +5 and became unresponsive to platelet transfusions but did respond to IVIG (6).

Finally, the most common BMT conditioning for GT has been BUCY with or without fludarabine. Non-ablative conditioning in one case was associated with graft rejection (1). Reduced intensity conditioning followed by matched related peripheral blood graft resulted in stable $30 \%$ whole blood donor chimerism, which was evidently sufficient to correct the bleeding disorder (7). Our two cases suggest that targeted busulfan, cyclophosphamide (BUCY), and antithymocyte globulin can be effective for achieving durable cord blood engraftment and correction of bleeding in GT. Full restoration of platelet GPIIb/IIIa expression is not required to correct the bleeding disorder as demonstrated when siblings with intermediate GPIIb/IIIa expression were marrow donors $(2,3)$. Nonetheless, a normal unrelated donor is more likely to 


\section{Cord blood transplantation for GT}

\section{fully restore GPIIb/IIIa expression as shown by} Patient 2 (Fig. 1).

Our two cases provide proof of principle that cord blood can be an appropriate stem cell source for HCT after targeted BUCY conditioning for children with life-threatening GT.

\section{Acknowledgment}

We thank Gayle Teramura, BS at Puget Sound Blood Center, Seattle, WA, for platelet antibody studies.

\section{References}

1. Bellucci S, Devergie A, Gluckman E, et al. Complete correction of Glanzmann's thrombasthenia by allogeneic bone-marrow transplantation. Br J Haematol 1985: 59: 635641.

2. Johnson A, Goodall AH, Downie CJ, Vellodi A, Michael DP. Bone marrow transplantation for Glanzmann's thrombasthenia. Bone Marrow Transplant 1994: 14: 147-150.

3. McColl MD, Gibson BES. Sibling allogeneic bone marrow transplantation in a patient with type I Glanzmann's thrombasthenia. Br J Haematol 1997: 99: 58-60.

4. Belluci S, Damaj G, Boval B, et al. Bone marrow transplantation in Glanzmann's thromasthenia with antiplatelet alloimmunization. Bone Marrow Transplant 2000: 25: 327-330.

5. Fuлimoto T-T, Kishimoto $\mathrm{M}$, Ide $\mathrm{K}$, et al. Glanzmann thrombasthenia with acute myeloid leukemia successfully treated by bone marrow transplantation. Int J Hematol 2005: 81: 77-80.

6. Flood VH, Johnson FL, Boshkov LK, et al. Sustained engraftment post bone marrow transplant despite anti-platelet antibodies in Glanzmann thrombasthenia. Pediatr Blood Cancer 2005: 45: 971-975.

7. Connor P, Khair K, Liesner R, et al. Stem cell transplantation for children with Glanzmann thrombasthenia. $\mathrm{Br} \mathrm{J}$ Haematol 2008: 140: 568-571.

8. Ishaqi MK, El-HayeK M, Gassas A, et al. Allogeneic stem cell transplantation for Glanzmann thrombasthenia. Pediatr Blood Cancer 2009: 52: 682-683.

9. Poon MC, D'olron R, HANn I, et al. Use of recombinant factor VIIa (NovoSeven) in patients with Glanzmann thrombasthenia. Semin Hematol 2001: 38: 21-25.

10. Parodi E, Rivetti E, Amendola G, et al. Long-term followup analysis after rituximab therapy in children with refractory symptomatic ITP: Identification of factors predictive of a sustained response. Br J Haematol 2009: 144: 552-558.

11. Cutler C, Miklos D, Kim HT, et al. Rituximab for steroidrefractory chronic graft-versus-host disease. Blood 2006: 108: 756-762.

12. Page KM, Mendizabal AM, Prasad VK, et al. Posttransplant autoimmune hemolytic anemia and other autoimmune cytopenias are increased in very young infants undergoing unrelated donor umbilical cord blood transplantation. Biol Blood Marrow Transplant 2008: 14: 1108-1117.

13. Vo AA, Lukovsky M, Toyoda $\mathrm{M}$, et al. Rituximab and intravenous immune globulin for desensitization during renal transplantation. N Engl J Med 2008: 359: 242-251.

14. Vieira CA, Agarwal A, Book BK, et al. Rituximab for reduction of anti-HLA antiboidies in patients awaiting renal transplantation: 1. Safety, pharmacodynamics, and pharmacokinetics. Transplantation 2004: 77: 542-548.

15. Sonnenday CJ, Warren DS, Cooper M, et al. Plasmapheresis, CMV hyperimmune globulin, and anti-CD20 allow ABOincompatible renal transplantation without splenectomy. Am J Transplant 2004: 4: 1315-1322. 